PROCEEDINGS OF THE

AMERICAN MATHEMATICAL SOCIETY

Volume 128, Number 3, Pages 793-801

S 0002-9939(99)05396-4

Article electronically published on September 9, 1999

\title{
COMPLETELY CONTINUOUS MULTILINEAR OPERATORS ON $C(K)$ SPACES
}

\author{
IGNACIO VILLANUEVA
}

(Communicated by Dale Alspach)

\begin{abstract}
Given a $k$-linear operator $T$ from a product of $C(K)$ spaces into a Banach space $X$, our main result proves the equivalence between $T$ being completely continuous, $T$ having an $X$-valued separately $\omega^{*}-\omega^{*}$ continuous extension to the product of the biduals and $T$ having a regular associated polymeasure. It is well known that, in the linear case, these are also equivalent to $T$ being weakly compact, and that, for $k>1, T$ being weakly compact implies the conditions above but the converse fails.
\end{abstract}

The purpose of this paper is to present some results concerning vector-valued completely continuous operators from a product of $C(K)$ spaces.

First we will explain our notation: if $K$ is a compact Hausdorff space, $C(K)$ will be the space of scalar-valued continuous functions on $K$ endowed with the supremun norm, $\Sigma$ will denote the $\sigma$-algebra of the Borel sets of $K$, and $B(\Sigma)$ will be the space of $\Sigma$-measurable functions on $K$ which are the uniform limit of $\Sigma$-simple functions. $X$ will denote a Banach space and $X^{* *}$ its bidual; we will assume, when necessary, that $X$ is embedded in $X^{* *}$. We shall use the convention.$i[$. to mean that the $i$-th coordinate is not involved. If $E_{1}, \ldots, E_{k}, X$ are Banach spaces, we will denote by $\mathcal{L}^{k}\left(E_{1}, \ldots, E_{k} ; X\right)$ the Banach space of the continuous multilinear operators from $E_{1} \times \cdots \times E_{k}$ into $X$ with the usual operator norm. As is well known, the Riesz representation theorem gives a representation of the operators on $C(K)$ as integrals with respect to Radon measures, and this has been very fruitfully used in the study of the properties of the $C(K)$ spaces and the operators defined on them. In a series of papers (see especially [9], [10]), Dobrakov developed a theory of polymeasures, functions defined on a product of $\sigma$-algebras which are measures on each variable separately, that can be used to obtain a Riesz-style representation theorem for multilinear operators defined on a product of $C(K)$ spaces.

We will denote the semivariation of a polymeasure $\gamma$ by $\|\gamma\|$ (for the general theory of polymeasures see [9] or [16]). It seems convenient to recall here that a polymeasure is called regular if it is separately regular and it is called countably additive if it is separately countably additive.

We now state the previously announced general representation theorem which extends and completes previous results (see [6]).

Received by the editors March 8, 1998 and, in revised form, April 24, 1998.

1991 Mathematics Subject Classification. Primary 46E15, 46B25.

Key words and phrases. $C(K)$ spaces, completely continuous, multilinear operators, AronBerner extension.

This work was partially supported by DGES grant PB97-0240. 
Theorem 1. Let $K_{1}, \ldots, K_{k}$ be compact Hausdorff spaces, let $X$ be a Banach space and let $T \in \mathcal{L}^{k}\left(C\left(K_{1}\right), \ldots, C\left(K_{k}\right) ; X\right)$. Then there is a unique

$$
\tilde{T} \in \mathcal{L}^{k}\left(B\left(\Sigma_{1}\right), \ldots, B\left(\Sigma_{k}\right), X^{* *}\right)
$$

which extends $T$ and is $\omega^{*}-\omega^{*}$ separately continuous (the $\omega^{*}$-topology that we consider in $B\left(\Sigma_{i}\right)$ is the one induced by the $\omega^{*}$-topology of $\left.C\left(K_{i}\right)^{* *}\right)$. In addition, we have

1. $\|T\|=\|\tilde{T}\|$.

2. For every $\left(g_{1},{ }^{[i]}, g_{k}\right) \in B\left(\Sigma_{1}\right) \times\left[{ }^{[i]} . \times B\left(\Sigma_{k}\right)\right.$ there is a unique $X^{* *}$-valued bounded $\omega^{*}$-Radon measure $\gamma_{g_{1},[i], g_{k}}$ on $K_{i}$ (i.e., an $X^{* *}$-valued finitely additive bounded vector measure on the Borel subsets of $K_{i}$, such that for every $x^{*} \in X^{*}$, $x^{*} \circ \gamma_{g_{1},[i], g_{k}}$ is a Radon measure on $\left.K_{i}\right)$, satisfying

$$
\int g_{i} d \gamma_{g_{1},[i], g_{k}}=\tilde{T}\left(g_{1}, \ldots, g_{i-1}, g_{i}, g_{i+1}, \ldots, g_{k}\right), \text { for all } g_{i} \in B\left(\Sigma_{i}\right) \text {. }
$$

3. $\tilde{T}$ is $\omega^{*}-\omega^{*}$ sequentially continuous (i.e., if $\forall i=1, \ldots, k,\left(g_{i}^{n}\right)_{n \in \mathbb{N}} \subset B\left(\Sigma_{i}\right)$, $g_{i}^{n} \stackrel{\omega^{*}}{\rightarrow} g_{i}$, then $\lim _{n \rightarrow \infty} \tilde{T}\left(g_{1}^{n}, \ldots, g_{k}^{n}\right)=\tilde{T}\left(g_{1}, \ldots, g_{k}\right)$ in the $\sigma\left(X^{* *}, X^{*}\right)$ topology $)$.

Also, if we define $\gamma: B\left(\Sigma_{1}\right) \times \cdots \times B\left(\Sigma_{k}\right) \mapsto X^{* *}$ by

$$
\gamma\left(A_{1}, \ldots, A_{k}\right)=\tilde{T}\left(\chi_{A_{1}}, \ldots \chi_{A_{k}}\right)
$$

then $\gamma$ is a polymeasure of bounded semivariation that satisfies

(a) $\|T\|=\|\gamma\|$.

(b) $T\left(f_{1}, \ldots, f_{k}\right)=\int\left(f_{1}, \ldots, f_{k}\right) d \gamma\left(f_{i} \in C\left(K_{i}\right)\right)$.

(c) For every $x^{*} \in X^{*}, x^{*} \circ \gamma$ is a separately regular polymeasure and the map $x^{*} \mapsto x^{*} \circ \gamma$ is continuous for the topologies $\sigma\left(X^{*}, X\right)$ and

$$
\sigma\left(\left(C\left(K_{1}\right) \hat{\otimes} \cdots \hat{\otimes} C\left(K_{k}\right)\right)^{*}, C\left(K_{1}\right) \hat{\otimes} \cdots \hat{\otimes} C\left(K_{k}\right)\right) .
$$

Conversely, if $\gamma: B\left(\Sigma_{1}\right) \times \cdots \times B\left(\Sigma_{k}\right) \mapsto X^{* *}$ is a polymeasure which satisfies (c), then it has finite semivariation and formula (b) defines a $k$-linear continuous operator from $C\left(K_{1}\right) \times \cdots \times C\left(K_{k}\right)$ into $X$ for which (a) holds.

Therefore the correspondence $T \leftrightarrow \gamma$ is an isometric isomorphism.

It is also proved in [6] that, if $E_{1}, \ldots, E_{k}$ are Banach spaces such that, for every $i \neq j$, every linear operator from $E_{i}$ into $E_{j}^{*}$ is weakly compact, then every operator $T \in \mathcal{L}^{k}\left(E_{1}, \ldots, E_{k} ; X\right)$ can be uniquely extended, with the same norm and separate $\omega^{*}-\omega^{*}$ continuity, to an operator $T^{* *} \in \mathcal{L}^{k}\left(E_{1}^{* *}, \ldots, E_{k}^{* *} ; X^{* *}\right)$. Of course the condition is fulfilled if every $E_{i}$ is a $C\left(K_{i}\right)$ space and in that case $\tilde{T}$ is the restriction of $T^{* *}$ to $B\left(\Sigma_{1}\right) \times \cdots \times B\left(\Sigma_{k}\right)$. It is worth noting that, in case all the $E_{i}$ 's are equal and $T$ is symmetric, $T^{* *}$ coincides with the well known Aron-Berner extension of $T$ (see [2], [1]).

It is known (see [6]) that if $T$ is weakly compact, then $T^{* *}$ is $X$-valued, although the reciprocal is not true in general. The main result in the present paper states that $T$ is completely continuous if and only if $T^{* *}$ is $X$-valued.

If $E_{1}, \ldots, E_{k}, X$ are Banach spaces, a multilinear operator $T \in \mathcal{L}^{k}\left(E_{1}, \ldots, E_{k} ; X\right)$ is said to be completely continuous if, given weakly Cauchy sequences $\left(x_{i}^{n}\right)_{n \in \mathbb{N}} \subset E_{i}$ $(i=1, \ldots, k)$, the sequence $\left(T\left(x_{1}^{n}, \ldots, x_{k}^{n}\right)\right)$ is norm convergent. These operators are studied, among other places, in [12], [13] and [15].

Our first result is a lemma crucial for the proof of the main results. 
Lemma 2. Let $T \in \mathcal{L}^{k}\left(C\left(K_{1}\right), \ldots, C\left(K_{k}\right) ; X\right)$. Then $T$ is completely continuous if and only if for all weak Cauchy sequences $\left(f_{j}^{n}\right)_{n \in \mathbb{N}} \subset C\left(K_{j}\right)(1 \leq j \leq k)$, and every $i \in 1, \ldots, k$, the measures $\left\{\gamma_{f_{1}^{n},[i], f_{k}^{n}} ; n \in \mathbb{N}\right\}$ mentioned in Theorem 1 are uniformly countably additive.

Proof. Let us first suppose $T$ to be completely continuous and let us fix $i$ with $1 \leq i \leq k$. We define the operators $T_{n} \in \mathcal{L}\left(C\left(K_{i}\right) ; X\right)$ by

$$
T_{n}\left(f_{i}\right)=T\left(f_{1}^{n}, \ldots, f_{i-1}^{n}, f_{i}, f_{i+1}^{n}, \ldots, f_{k}\right) .
$$

Since $T$ is completely continuous, we get that for each $n \in \mathbb{N}, T_{n}$ is also completely continuous. By the reciprocal Dunford-Pettis property of $C(K)$ this means that $T_{n}$ is weakly compact and therefore its associated measure $\gamma_{n}=\gamma_{f_{1}^{n},[i], f_{k}^{n}}$ is countably additive and regular.

Now, were the measures $\left(\gamma_{n}\right)_{n \in \mathbb{N}}$ not uniformly countably additive, there would exist an $\epsilon>0$ and a sequence $\left(A_{i}^{m}\right)_{m \in \mathbb{N}}$ of disjoint open sets of $\Sigma_{i}$ such that for all $m \in \mathbb{N}$,

$$
\sup _{n}\left\|\gamma_{n}\left(A_{i}^{m}\right)\right\|>\epsilon
$$

Then we could choose two sequences of indexes $(m(p))_{p \in \mathbb{N}},(n(p))_{p \in \mathbb{N}}$, where $(n(p))$ is an increasing sequence, such that

$$
\left\|\gamma_{n(p)}\left(A_{i}^{m(p)}\right)\right\|>\epsilon .
$$

For simplicity we will write this as

$$
\left\|\gamma_{p}\left(A_{i}^{p}\right)\right\|>\epsilon .
$$

Since every $\gamma_{n}$ is regular, we see that for each $p \in \mathbb{N}$ there would exist an $f_{i}^{p} \in C\left(K_{i}\right)$ such that $\operatorname{supp} f_{i}^{p} \subset A_{i}^{p},\left\|f_{i}^{p}\right\| \leq 1$, and

$$
\left\|\int f_{i}^{p} d \gamma_{p}\right\|>\epsilon, \text { i.e., }\left\|T\left(f_{1}^{p}, \ldots, f_{i}^{p}, \ldots, f_{k}^{p}\right)\right\|>\epsilon ;
$$

but clearly $f_{i}^{p}$ weakly converges to 0 and therefore, $T$ being completely continuous,

$$
\left\|T\left(f_{1}^{p}, \ldots, f_{i}^{p}, \ldots, f_{k}^{p}\right)\right\| \rightarrow 0
$$

(the proof of this fact can be found in Lemma 2.4 and Theorem 2.3 in [4]), a contradiction.

For the other implication, let us choose for every $j=1, \ldots, k,\left(f_{j}^{n}\right) \subset C\left(K_{j}\right)$ to be weakly Cauchy sequences, with $\left\|f_{j}^{n}\right\| \leq 1$, such that at least one of them, say $\left(f_{i}^{n}\right)$, weakly converges to 0 . According to our hypothesis, the measures $\left\{\gamma_{n}=\right.$ $\left.\gamma_{f_{1}^{n},[i], f_{k}^{n}} ; n \in \mathbb{N}\right\}$ are uniformly countably additive. Let $\lambda$ be a positive measure such that the measures $\left(\gamma_{n}\right)_{n \in \mathbb{N}}$ are uniformly $\lambda$-continuous. For every $\epsilon>0$ there exists a $\delta>0$ such that

$$
\sup _{n \in \mathbb{N}}\left\|\gamma_{n}(A)\right\|<\frac{\epsilon}{2} \text { when } \lambda(A)<\delta .
$$

Since $f_{i}^{n} \stackrel{\omega}{\rightarrow} 0$, then $\forall t \in K_{i}, f_{i}^{n}(t) \rightarrow 0$. Then, according to Egoroff's theorem, there is a compact $K_{i}^{\prime} \subset K_{i}$ such that $f_{i}^{n} \rightarrow 0$ uniformly on $K_{i}^{\prime}$ and $\lambda\left(K_{i} \backslash K_{i}^{\prime}\right)<\delta$. Let $n_{0} \in \mathbb{N}$ be such that for every $n>n_{0}$,

$$
\left\|f_{i}^{n}\right\|_{K_{i}^{\prime}} \leq \frac{\epsilon}{2\|\gamma\|} \text {, where }\|f\|_{K_{i}^{\prime}}=\sup _{t \in K_{i}^{\prime}} f(t) .
$$


Then, for every $n>n_{0}$,

$$
\begin{gathered}
\left\|T\left(f_{1}^{n}, \ldots, f_{k}^{n}\right)\right\|=\left\|\int_{K_{i}} f_{i}^{n} d \gamma_{n}\right\| \leq\left\|\int_{K_{i}^{\prime}} f_{i}^{n} d \gamma_{n}\right\|+\left\|\int_{K_{i} \backslash K_{i}^{\prime}} f_{i}^{n} d \gamma_{n}\right\| \\
\leq\left\|f_{i}^{n}\right\|_{K_{i}^{\prime}}\left\|\gamma_{n}\right\|\left(K_{i}^{\prime}\right)+\left\|\gamma_{n}\right\|\left(K_{i} \backslash K_{i}^{\prime}\right)<\frac{\epsilon}{2}+\frac{\epsilon}{2}=\epsilon .
\end{gathered}
$$

Therefore

$$
\left\|T\left(f_{1}^{n}, \ldots, f_{k}^{n}\right)\right\| \rightarrow 0 .
$$

The hypothesis that $f_{i}^{n} \stackrel{\omega}{\rightarrow} 0$ can now be removed in a standard way (see [15], end of the proof of Theorem 2.1) and we conclude that $T$ is completely continuous.

We will also use another result, whose proof can be found in [6, Corollary 4].

Proposition 3. Let $T \in \mathcal{L}^{k}\left(C\left(K_{1}\right), \ldots, C\left(K_{k}\right) ; X\right)$. With the notations of Theorem 1 , if $\tilde{T}$ is $X$-valued, then $\tilde{T}$ is sequentially $\omega^{*}-\|\cdot\|$ continuous.

We also need the following lemma from [15].

Lemma 4. Let $E, X$ be Banach spaces and let $T \in \mathcal{L}\left(E ; c_{0}(X)\right)$, where $T(x)=$ $\left(T_{n}(x)\right)_{n}$. Then $T$ is weakly compact if and only if

i) for every $n \in \mathbb{N}, T_{n} \in \mathcal{L}(E ; X)$ is weakly compact, and

ii) for every $z \in E^{* *}, \lim _{n}\left\|T_{n}^{* *}(z)\right\|=0$.

Before stating our main result we must observe that, given an operator $T \in$ $\mathcal{L}^{k}\left(C\left(K_{1}\right), \ldots, C\left(K_{k}\right) ; X\right)$, the fact that $T^{* *}$ is separately $\omega^{*}-\omega^{*}$ continuous implies that, for every $\left(f_{1}, \ldots, f_{k-1}\right) \in C\left(K_{1}\right) \times \cdots \times C\left(K_{k-1}\right)$ and for every $z_{k} \in C\left(K_{k}\right)^{* *}$,

$$
\left(T_{f_{1}, \ldots, f_{k-1}}\right)^{* *}\left(z_{k}\right)=T^{* *}\left(f_{1}, \ldots, f_{k-1}, z_{k}\right)
$$

where $T_{f_{1}, \ldots, f_{k-1}} \in \mathcal{L}\left(C\left(K_{k}\right) ; X\right)$ is the operator defined as

$$
T_{f_{1}, \ldots, f_{k-1}}\left(f_{k}\right)=T\left(f_{1}, \ldots, f_{k-1}, f_{k}\right)
$$

and $\left(T_{f_{1}, \ldots, f_{k-1}}\right)^{* *}$ is its bitranspose. The same fact also implies that

$$
\left(T_{z_{k}}\right)^{* *}=\left(T^{* *}\right)_{z_{k}}
$$

where $T_{z_{k}} \in \mathcal{L}^{k-1}\left(C\left(K_{1}\right), \ldots, C\left(K_{k-1}\right) ; X\right)$ is the operator defined as

$$
T_{z_{k}}\left(f_{1}, \ldots, f_{k-1}\right)=T^{* *}\left(f_{1}, \ldots, f_{k-1}, z_{k}\right)
$$

and $\left(T^{* *}\right)_{z_{k}} \in \mathcal{L}^{k-1}\left(C\left(K_{1}\right)^{* *}, \ldots, C\left(K_{k-1}\right)^{* *} ; X\right)$ is the operator defined as

$$
\left(T^{* *}\right)_{z_{k}}\left(z_{1}, \ldots, z_{k-1}\right)=T^{* *}\left(z_{1}, \ldots, z_{k-1}, z_{k}\right) .
$$

Both of these equalities are used in the proof of the next theorem.

We are now ready to state our main result. Part of its proof is a modification of an idea in [11] which in turn is based on [15].

Theorem 5. Let $K_{1}, \ldots, K_{k}$ be compact Hausdorff spaces, let $X$ be a Banach space and let $T \in \mathcal{L}^{k}\left(C\left(K_{1}\right), \ldots, C\left(K_{k}\right) ; X\right)$. Then, using the notations of Theorem 1 , the following are equivalent:

a) $T$ is completely continuous.

b) $T^{* *}$ is $X$-valued.

c) $\tilde{T}$ is $X$-valued.

d) $\gamma$ is countably additive.

e) $\gamma$ is regular. 
Proof. a) $\Rightarrow$ b): We will argue by induction on $k$. If $k=1$, the result is well known. Suppose it is true for $k-1$. Now let $g$ belong to $C\left(K_{k}\right)^{* *}$. We define the operator $T_{g} \in \mathcal{L}^{k-1}\left(C\left(K_{1}\right) \times \cdots \times C\left(K_{k-1}\right) ; X\right)$ as

$$
T_{g}\left(f_{1}, \ldots, f_{k-1}\right)=T^{* *}\left(f_{1}, \ldots, f_{k-1}, g\right) .
$$

To check that $T_{g}$ is indeed $X$-valued, let us observe that if we fix $\left(f_{1}, \ldots, f_{k-1}\right) \in$ $C\left(K_{1}\right) \times \cdots \times C\left(K_{k-1}\right)$, the operator $T_{f_{1}, \ldots, f_{k-1}} \in \mathcal{L}\left(C\left(K_{k}\right) ; X\right)$ defined as

$$
T_{f_{1}, \ldots, f_{k-1}}(f)=T\left(f_{1}, \ldots, f_{k-1}, f\right)
$$

is completely continuous, therefore also weakly compact, and hence $\left(T_{f_{1}, \ldots, f_{k-1}}\right)^{* *}$ is $X$-valued. Since $\forall g \in C\left(K_{k}\right)^{* *}$,

$$
\left(T_{f_{1}, \ldots, f_{k-1}}\right)^{* *}(g)=T_{g}\left(f_{1}, \ldots, f_{k-1}\right),
$$

we obtain that $T_{g}$ is $X$-valued.

$T_{g}$ is clearly linear and continuous. We will prove now that it is also completely continuous: for this, let us consider $\left(\left(f_{1}^{n}\right), \ldots,\left(f_{k-1}^{n}\right)\right) \subset C\left(K_{1}\right) \times \cdots \times C\left(K_{k-1}\right)$ to be weakly Cauchy sequences, with $\left\|f_{i}^{n}\right\| \leq 1$, at least one of which weakly converges to zero. To simplify notation we assume $f_{1}^{n} \stackrel{\omega}{\rightarrow} 0$. Then, if we fix $f_{k} \in$ $C\left(K_{k}\right)$, the sequence $\left(\left\|T\left(f_{1}^{n}, \ldots, f_{k-1}^{n}, f_{k}\right)\right\|\right)_{n}$ converges to zero, as can again be seen in [4], Lemma 2.4 and Theorem 2.3. Therefore we can define the operator $S \in \mathcal{L}\left(C\left(K_{k}\right) ; c_{0}(X)\right)$ as

$$
S(f)=\left(T\left(f_{1}^{n}, \ldots, f_{k-1}^{n}, f_{k}\right)\right)_{n \in \mathbb{N}}=\left(T_{n}\left(f_{k}\right)\right)_{n \in \mathbb{N}}
$$

$S$ is obviously linear and continuous. We will show now that $S$ is completely continuous: let $\left(f_{k}^{m}\right) \subset C\left(K_{k}\right)$ be a weakly converging to zero sequence. We want to prove that $S\left(f_{k}^{m}\right) \rightarrow 0$, i.e., that

$$
\sup _{n \in \mathbb{N}}\left\|T\left(f_{1}^{n}, \ldots, f_{k-1}^{n}, f_{k}^{m}\right)\right\| \stackrel{m}{\rightarrow} 0 .
$$

We must recall here that, with the notation of Theorem 1, 2,

$$
T\left(f_{1}^{n}, \ldots, f_{k-1}^{n}, f_{k}^{m}\right)=\int f_{k}^{m} d \gamma_{f_{1}^{n}, \ldots, f_{k-1}^{n}} .
$$

According to Lemma 2, the measures $\gamma_{n}=\gamma_{f_{1}^{n}, \ldots, f_{k-1}^{n}}$ are uniformly countably additive. Take $\epsilon>0$. Similarly as in the "if" part of Lemma 2, we can obtain, for all $n$ and for $m$ large enough, $\left\|T\left(f_{1}^{n}, \ldots, f_{k-1}^{n}, f_{k}^{m}\right)\right\|<\epsilon$.

Hence, $S$ is completely continuous. Then $S$ is also weakly compact and, according to Lemma 4 , this implies that for every $g \in C\left(K_{k}\right)^{* *}$,

$$
\lim _{n \rightarrow \infty}\left\|T_{n}^{* *}(g)\right\|=0 .
$$

Since $T_{n}^{* *}(g)=T^{* *}\left(f_{1}^{n}, \ldots, f_{k-1}^{n}, g\right)=T_{g}\left(f_{1}^{n}, \ldots, f_{k-1}^{n}\right)$ we get that

$$
\lim _{n \rightarrow \infty}\left\|T_{g}\left(f_{1}^{n}, \ldots, f_{k-1}^{n}\right)\right\|=0 .
$$

As in Lemma 2, we conclude that $T_{g}$ is completely continuous. Now the induction hypothesis tells us that $T_{g}^{* *}$ is $X$-valued. Since this happens for every $g \in C(K)^{* *}$, we get that $T^{* *}$ is $X$-valued.

b) $\Rightarrow$ c) is obvious, and c) $\Rightarrow$ a) is a direct consequence of Proposition 3 above.

c) $\Rightarrow$ d) follows either from Proposition 3 or from the Orlicz-Pettis theorem. To see that $\mathrm{d}) \Rightarrow \mathrm{e}$ ) we only need to consider that $\gamma$ is separately countably additive and separately $\omega^{*}$-regular, therefore separately regular. e) $\Rightarrow$ d) follows from the Alexandroff theorem. 
d) $\Rightarrow \mathrm{c}$ ): First we observe that if $\gamma$ is countably additive, so are the measures $\gamma_{g_{1},[i], g_{k}}$ defined in Theorem 1,2 . This is obvious when, for every $j \in\{1,[i] ., k\}, g_{j}$ is a $\Sigma_{j}$-simple function, and it follows easily for general $g_{j}$ just considering that the $\Sigma_{j}$ simple functions are dense in $B\left(\Sigma_{j}\right)$ and using the Vitali-Hahn-Saks-Nikodým theorem. Let us fix now $f_{2}, \ldots, f_{k} \in C\left(K_{2}\right) \times \cdots \times C\left(K_{k}\right) \cdot \gamma_{f_{2}, \ldots, f_{k}}$ is the Radon measure associated to the operator $T_{f_{2}, \ldots, f_{k}} \in \mathcal{L}\left(C\left(K_{1}\right) ; X\right)$ defined as $T_{f_{2}, \ldots, f_{k}}\left(f_{1}\right)=$ $T\left(f_{1}, f_{2}, \ldots, f_{k}\right)$. It is then well known that the fact that $\gamma_{f_{2}, \ldots, f_{k}}$ is countably additive implies that $\left(T_{f_{2}, \ldots, f_{k}}\right)^{* *}$ is $X$-valued, and, therefore, $\tilde{T}\left(g_{1}, f_{2}, \ldots, f_{k}\right) \in X$ for all $g_{1} \in B\left(\Sigma_{1}\right)$. Next we consider the operator $T_{g_{1}, f_{3}, \ldots, f_{k}} \in \mathcal{L}\left(C\left(K_{2}\right) ; X\right)$ defined as $T_{g_{1}, f_{3}, \ldots, f_{k}}\left(f_{2}\right)=\tilde{T}\left(g_{1}, f_{2}, f_{3}, \ldots, f_{k}\right)$. By analogous reasonings we obtain that $\tilde{T}\left(g_{1}, g_{2}, f_{3}, \ldots, f_{k}\right) \in X$ for all $\left(g_{1}, g_{2}\right) \in B\left(\Sigma_{1}\right) \times B\left(\Sigma_{2}\right)$. Proceeding likewise we finish the proof.

The equivalence between c), d) and e) above can be found in [10, Theorem 6], where only the Baire $\sigma$-algebras are considered. The reasonings in that paper do not apply to Borel $\sigma$-algebras.

It is already known (see [14, p. 385]) that, contrary to what happens in the linear case, for $k>1$ there exist completely continuous $k$-homogeneous polynomials on $C(K)$ which are not weakly compact; we want to point out that our result above seems to indicate that in the case of polynomials and multilinear operators, at least on $C(K)$, the class of the completely continuous operators could somehow be the "right" class for certain applications.

Corollary 5 from [6] states that if $E_{1}, \ldots, E_{k}$ are Banach spaces such that, for every $i \neq j$ every linear operator $E_{i}$ into $E_{j}^{*}$ is weakly compact and in addition, for every $i=1, \ldots, k$, every linear operator from $E_{i}$ into $X$ is weakly compact (if all the $E_{i}$ 's are $C\left(K_{i}\right)$ spaces, this is the case, for example, if $X \not \supset c_{0}$, or if every $K_{i}$ is stonean and $X \not \supset l_{\infty}$, or if every $C\left(K_{i}\right)$ is a Grothendieck space and $X$ is separable), then the extension $T^{* *}$ mentioned after Theorem 1 of any operator $T \in \mathcal{L}^{k}\left(E_{1}, \ldots, E_{k} ; X\right)$ is $X$-valued. Using that, we get the next corollary (see [11, Corollary 7 ] where the result is stated, in the polynomial case, in a more general setting).

Corollary 6. Let $K_{1}, \ldots, K_{k}$ be compact Hausdorff spaces, and $X$ a Banach space. If every linear operator from every $C\left(K_{i}\right)$ into $X$ is weakly compact, then every multilinear operator $T \in \mathcal{L}^{k}\left(C\left(K_{1}\right), \ldots, C\left(K_{k}\right) ; X\right)$ is completely continuous.

We will now prove a strengthening of Lemma 2 which will be necessary for the proof of the next proposition.

Lemma 7. Let $T \in \mathcal{L}^{k}\left(C\left(K_{1}\right), \ldots, C\left(K_{k}\right) ; X\right)$, and for $i=1, \ldots, k$ let $\tilde{T}_{i} \in$ $\mathcal{L}^{k}\left(B\left(\Sigma_{1}\right), \ldots, B\left(\Sigma_{i}\right), C\left(K_{i+1}\right), \ldots, C\left(K_{k}\right) ; X\right)$ be the restriction of $\tilde{T}$ to $B\left(\Sigma_{1}\right) \times$ $\cdots \times B\left(\Sigma_{i}\right) \times C\left(K_{i+1}\right) \times \cdots \times C\left(K_{k}\right)$. For $j=1, \ldots, i$ let $\left(g_{j}^{n}\right)_{n \in \mathbb{N}} \subset B\left(\Sigma_{j}\right)$ and for $j=i+1, \ldots, k$ let $\left(g_{j}^{n}\right)_{n \in \mathbb{N}} \subset C\left(K_{j}\right)$ be weakly Cauchy sequences. If $\tilde{T}_{i}$ is completely continuous, then, for every $l=1, \ldots, k$, the measures $\left\{\gamma_{g_{1}^{n},[!], g_{k}^{n}} ; n \in \mathbb{N}\right\}$ are uniformly countably additive.

Proof. We only need to realize that in that case $T$ is completely continuous, and therefore, according to Theorem 5, the measures $\left\{\gamma_{g_{1}^{n},[l], g_{k}^{n}} ; n \in \mathbb{N}\right\}$ are $X$-valued and countably additive. Now the proof proceeds exactly like the proof of Lemma 2 . 
The next result is an application of Theorem 5 .

Proposition 8. Let $K_{1}, \ldots, K_{k}$ be compact Hausdorff spaces, $X$ a Banach space, $T \in \mathcal{L}^{k}\left(C\left(K_{1}\right), \ldots, C\left(K_{k}\right) ; X\right)$ and $\tilde{T}$ its extension defined in Theorem 1 . Then $T$ is completely continuous if and only if $\tilde{T}$ is completely continuous.

Proof. One of the implications is clear. For the other, let us suppose $T$ as in the hypothesis, and let us define $\tilde{T}_{1}$ as the restriction of $\tilde{T}$ to $B\left(\Sigma_{1}\right) \times C\left(K_{2}\right) \times \cdots \times$ $C\left(K_{k}\right)$. First we want to prove that $\tilde{T}_{1}$ is completely continuous. Let $\left(g_{1}^{n}\right) \subset B\left(\Sigma_{1}\right)$, $\left(f_{2}^{n}\right) \subset C\left(K_{2}\right), \ldots,\left(f_{k}^{n}\right) \subset C\left(K_{k}\right)$ be weakly Cauchy sequences, all of them in the respective unit ball. All we need to prove is that, if any one of the $k$ weakly Cauchy sequences above chosen weakly converges to 0 , then

$$
\lim _{n \rightarrow \infty}\left\|\tilde{T}_{1}\left(g_{1}^{n}, f_{2}^{n}, \ldots, f_{k}^{n}\right)\right\|=0 .
$$

The proof of this fact is slightly different depending on whether the chosen sequence is the first one or any of the others. In the first case, Lemma 7 or Lemma 2 tells us that the measures $\left(\gamma_{n}=\gamma_{\left.f_{2}^{n}, \ldots, f_{k}^{n}\right)}\right)$ are uniformly countably additive. Let $\lambda$ be a positive measure such that the measures $\left(\gamma_{n}\right)_{n \in \mathbb{N}}$ are uniformly $\lambda$-continuous. For every $\epsilon>0$ there is a $\delta>0$ such that

$$
\left\|\gamma_{n}\right\|(A)<\epsilon / 3 \text { whenever } \lambda(A)<\delta .
$$

According to Luzin's theorem, there exists $K_{1}^{\prime} \subset K_{1}$ such that $\forall n \in \mathbb{N}$,

$$
g_{\left.1\right|_{K_{1}^{\prime}}}^{n}=f_{1}^{n} \in C\left(K_{1}^{\prime}\right) \text { and } \lambda\left(K_{1} \backslash K_{1}^{\prime}\right)<\delta .
$$

Let $H=\overline{\left[\left(f_{1}^{n}\right)_{n \in \mathbb{N}}\right]} \subset C\left(K_{1}^{\prime}\right)$. Theorem 1 in [5] tells us that there exists an extension operator $S: H \mapsto C\left(K_{1}\right)$; let us call $S\left(f_{1}^{n}\right)=h_{1}^{n}$. Since $\left(g_{1}^{n}\right)$ weakly converges to 0 , so do $\left(f_{1}^{n}\right)$ and $\left(h_{1}^{n}\right)$. This last fact allows us to choose an $n_{0} \in \mathbb{N}$ such that for every $n>n_{0}$,

$$
\left\|T\left(h_{1}^{n}, f_{2}^{n}, \ldots, f_{k}^{n}\right)\right\|<\epsilon / 3
$$

Then, for every $n>n_{0}$,

$$
\begin{gathered}
\left\|\tilde{T}\left(g_{1}^{n}, f_{2}^{n}, \ldots, f_{k}^{n}\right)\right\|=\left\|\int_{K_{1}} g_{1}^{n} d \gamma_{n}\right\| \\
=\left\|\int_{K_{1}^{\prime}} g_{1}^{n} d \gamma_{n}+\int_{K_{1} \backslash K_{1}^{\prime}} g_{1}^{n} d \gamma_{n}=\right\| \int_{K_{1}^{\prime}} h_{1}^{n} d \gamma_{n}+\int_{K_{1} \backslash K_{1}^{\prime}} g_{1}^{n} d \gamma_{n} \| \\
=\left\|\int_{K_{1}^{\prime}} h_{1}^{n} d \gamma_{n}+\int_{K_{1} \backslash K_{1}^{\prime}} h_{1}^{n} d \gamma_{n}-\int_{K_{1} \backslash K_{1}^{\prime}} h_{1}^{n} d \gamma_{n}+\int_{K_{1} \backslash K_{1}^{\prime}} g_{1}^{n} d \gamma_{n}\right\| \\
=\left\|\int_{K_{1}} h_{1}^{n} d \gamma_{n}-\int_{K_{1} \backslash K_{1}^{\prime}} h_{1}^{n} d \gamma_{n}+\int_{K_{1} \backslash K_{1}^{\prime}} g_{1}^{n} d \gamma_{n}\right\|\left\|_{K_{1} \backslash K_{1}^{\prime}} h_{1}^{n} d \gamma_{n}\right\| \int_{K_{1} \backslash K_{1}^{\prime}} g_{1}^{n} d \gamma_{n} \|<\epsilon .
\end{gathered}
$$

Now, if the sequence which weakly converges to 0 is not the first but any of the others, for example $f_{2}^{n}$, we consider again an $\epsilon>0$ and $\lambda, \delta, K_{1}^{\prime}, f_{1}^{n}$ and $h_{1}^{n}$ to be 
as previously defined. In this case $\left(h_{1}^{n}\right)$ does not in general weakly converge to 0 , but $\left(f_{2}^{n}\right)$ does, so again we can choose an $n_{0} \in \mathbb{N}$ such that for every $n>n_{0}$,

$$
\left\|T\left(h_{1}^{n}, f_{2}^{n}, \ldots, f_{k}^{n}\right)\right\|<\epsilon / 3
$$

Reasoning now as before we get again that if $n>n_{0}$,

$$
\left\|\tilde{T}\left(g_{1}^{n}, f_{2}^{n}, \ldots, f_{k}^{n}\right)\right\|<\epsilon
$$

This finishes the proof that $\tilde{T}_{1}: B\left(\Sigma_{1}\right) \times C\left(K_{2}\right) \times \cdots \times C\left(K_{k}\right) \mapsto X$ is completely continuous. We now consider $\tilde{T}_{2}$ as the restriction of $\tilde{T}$ to $B\left(\Sigma_{1}\right) \times B\left(\Sigma_{2}\right) \times C\left(K_{3}\right) \times$ $\cdots \times C\left(K_{k}\right)$. To prove that $\tilde{T}_{2}$ is also completely continuous we consider weakly Cauchy sequences $\left(g_{1}^{n}\right) \subset B\left(\Sigma_{1}\right),\left(g_{2}^{n}\right) \subset B\left(\Sigma_{2}\right),\left(f_{3}^{n}\right) \subset C\left(K_{3}\right), \ldots,\left(f_{k}^{n}\right) \subset C\left(K_{k}\right)$.

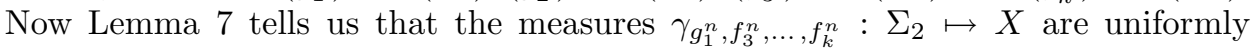
countably additive, and we can repeat almost exactly the previous reasonings to prove that $\tilde{T}_{2}$ is completely continuous. Proceeding likewise we finish the proof.

A couple of comments are probably appropriate here: first, let us recall that $\mathcal{L}^{k}\left(E_{1}, \ldots, E_{k} ; X\right)$ is isometrically isomorphic to $\mathcal{L}\left(E_{1} \hat{\otimes} \cdots \hat{\otimes} E_{k} ; X\right)$, where $E_{1} \hat{\otimes} \cdots$ $\hat{\otimes} E_{k}$ is the projective tensor product of $E_{1}, \ldots, E_{k}$. Given an operator $T \in$ $\mathcal{L}^{k}\left(E_{1}, \ldots, E_{k} ; X\right)$, let us denote by $\hat{T}$ the linear operator associated to it by the previously mentioned isomorphism. Then a natural question arises in this context: is it true that $T$ is completely continuous if and only if so is $\hat{T}$ ? It is easy to see that, for spaces with the Dunford-Pettis Property (in particular for $C(K)$ spaces), if $\hat{T}$ is completely continuous, then so is $T$. This follows from a well known result (see [8]) that states that if $E_{1}, \ldots, E_{k}$ are spaces with the Dunford-Pettis Property and $\left(x_{1}^{n}\right) \subset E_{1}, \ldots,\left(x_{k}^{n}\right) \subset E_{k}$ are weak Cauchy sequences, then the sequence $\left(x_{1}^{n} \otimes \cdots \otimes x_{k}^{n}\right) \subset E_{1} \hat{\otimes} \cdots \hat{\otimes} E_{k}$ is also weak Cauchy. For $C(K)$ spaces with $K$ scattered (equivalently $C(K)$ does not contain an isomorphic copy of $\ell_{1}$ ), the reciprocal is also true: if $T \in \mathcal{L}^{k}\left(C\left(K_{1}\right), \ldots, C\left(K_{k}\right) ; X\right)$ is completely continuous, then $T$ is weakly compact (see [12]). This, in turn, is equivalent to $\hat{T}$ being weakly compact, and since, for $K_{1}, \ldots, K_{k}$ scattered, $\left(C\left(K_{1}\right) \hat{\otimes} \cdots \hat{\otimes} C\left(K_{k}\right)\right)^{*}$ is Schur, we get that $C\left(K_{1}\right) \hat{\otimes} \cdots \hat{\otimes} C\left(K_{k}\right)$ has the Dunford-Pettis Property, which implies that $\hat{T}$ is completely continuous. For general $K$ it seems likely that there exist a Banach space $X$ and a completely continuous operator $T \in \mathcal{L}^{k}\left(C\left(K_{1}\right), \ldots, C\left(K_{k}\right) ; X\right)$ such that its

associated linear operator $\hat{T}$ is not completely continuous. Let us note that if this was not the case, then $C\left(K_{1}\right) \hat{\otimes} \cdots \hat{\otimes} C\left(K_{k}\right)$ would always have the Dunford-Pettis Property, and this is at the present time not known, for example for $C[0,1] \hat{\otimes} C[0,1]$ or for $\ell_{\infty} \hat{\otimes} \ell_{\infty}($ see $[7])$.

One final remark: in [3] a new and interesting class of multilinear scalar operators called regular operators (regularity which has no relation with the measure-theoretic notion of regularity of a measure or polymeasure) is defined and studied. In $C(K)$ spaces this class is contained in the class of the completely continuous multilinear scalar operators. If $K$ is scattered, both classes coincide, otherwise the containment is strict.

\section{ACKNOWLEDGEMENT}

The author would like to thank Fernando Bombal for his continuous help and encouragement and Joaquín Gutiérrez for his very valuable hints and suggestions. 


\section{REFERENCES}

[1] R. M. Aron, and P.D. Berner, A Hahn-Banach extension theorem for analytic mappings. Bull. Soc. Math. France, 106 (1978), 3-24. MR 80e:46029

[2] R. M. Aron, B. J. Cole, and T. W. Gamelin, Spectra of algebras of analytic functions on a Banach space.J. reine angew. Math., 415 (1991), 51-93. MR 92f:46056

[3] R. M. Aron and P. Galindo, Weakly compact multilinear mappings, Proc. Edinburgh Math. Soc., 40 (1997), 181-192. MR 98e:46051

[4] R. M. Aron, C. Hervés, and M. Valdivia, Weakly continuous mappings on Banach spaces.J. Funct. Anal., 52 (1983), 189-204. MR 84g:46066

[5] F. Bombal, and P. Cembranos, Characterization of some classes of operators on spaces of vector-valued continuous functions. Math. Proc. Cambridge Phil. Soc., 97 (1985), 137-146. MR 86b:47051

[6] F. Bombal, and I. Villanueva, Multilinear operators in spaces of continuous functions. Funct. Approx. Comment. Math. XXVI (1998), 117-126. CMP 99:07

[7] J. Castillo and M. Gonzalez, On the Dunford-Pettis Property. Acta Univ. Carol. Math. Phys., 35 (1994), 5-12. MR 97a:46018

[8] J. Diestel, A survey of results related to the Dunford-Pettis Property, in W. H. Graves (Ed.) Proc. Conf. on Integration, Topology and Geometry in linear spaces, Contemp. Math. 2 (1980), Amer. Math. Soc., Providence, R.I., pp. 15-60. MR 82i:46023

[9] I. Dobrakov, On integration in Banach spaces, VIII (polymeasures). Czech. Math. J., 37 (112) (1987), 487-506. MR 89a:46097

[10] I. Dobrakov, Representation of multilinear operators on $\times C_{0}\left(T_{i}\right)$. Czech. Math. J., 39 (114) (1989), 288-302. MR 90c: 46055

[11] M. González and J. Gutiérrez, When every polynomial is unconditionally converging. Arch. Math., 63 (1994), 145-151. MR 95h:46070

[12] M. González and J. Gutiérrez, Unconditionally converging polynomials on Banach spaces. Math. Proc. Cambridge Philos. Soc., 117 (1995), 321-331. MR 95j:46050

[13] A. Pełczyński, On weakly compact polynomial operators on B-spaces with Dunford-Pettis property. Bull. Acad. Polon. Sci. Ser. Sci. Math. Astr. Phys., 11 (1963), 371-378. MR 28:4369

[14] A. Pełczyński, A theorem of Dunford-Pettis type for polynomial operators. Bull. Acad. Polon. Sci. Ser. Sci. Math. Astr. Phys., 11 (1963), 379-386. MR 28:4370

[15] R. A. Ryan, Dunford-Pettis properties Bull. Acad. Polon. Sci. Ser. Sci. Math., 27 (1979), 373-379. MR 80m:46018

[16] I. Villanueva, Polimedidas y representación de operadores multilineales de $C\left(\Omega_{1}, X_{1}\right) \times \cdots \times$ $C\left(\Omega_{d}, X_{d}\right)$. Tesina de Licenciatura, Dpto. de Análisis Matemático, Fac. de Matemáticas, Universidad Complutense de Madrid, (1997).

Departamento de Análisis Matemático, Facultad de Matemáticas, Universidad ComPLUTENSE DE MADRID, 28040 MADRID, SPAIN

E-mail address: Ignacio_Villanueva@mat.ucm.es 